\title{
Design of RCS Reduction Applied to W-Band Circularly Polarized Antenna Array
}

\author{
Chun-Hong Chen $\left(\mathbb{D}\right.$, Pei-Yang Wang $\left(\mathbb{D}\right.$, Jun Chen $\left(\mathbb{D}\right.$, and Ting Xu ${ }^{(\mathbb{C}}$ \\ Nanjing University of Science and Technology, Nanjing, Jiangsu 210000, China \\ Correspondence should be addressed to Pei-Yang Wang; wpy@njust.edu.cn
}

Received 26 April 2021; Accepted 3 September 2021; Published 20 September 2021

Academic Editor: Chien-Jen Wang

Copyright (c) 2021 Chun-Hong Chen et al. This is an open access article distributed under the Creative Commons Attribution License, which permits unrestricted use, distribution, and reproduction in any medium, provided the original work is properly cited.

\begin{abstract}
A single-layer capsule-shaped polarization conversion metasurface (PCM) is proposed in this paper. In the W-band, its polarization conversion rate (PCR) exceeds $97 \%$, effectively changing the polarization direction of the incident wave. PCM is arranged in a chessboard array to achieve broadband RCS reduction. Placing the PCM array on a circularly polarized sequentially rotated slot antenna array, simulated results show that the radiation characteristics of the antenna array are hardly affected by the PCM array. The results of measurement demonstrate that the RCS of the antenna array with PCM array proposed is reduced by more than $10 \mathrm{~dB}$ from 40 to $119 \mathrm{GHz}$; the relative bandwidth $(-10 \mathrm{~dB})$ reaches $96.3 \%$.
\end{abstract}

\section{Introduction}

Modern battlefields require radars to have strong stealth capabilities; the most effective method is to reduce the radar cross-section (RCS). Slot antenna arrays are one of the commonly used antennas for millimeter wave radars, and their flat ground planes will produce strong scattering contributions in the vertical direction. Hence, it is of great significance to study the RCS reduction of slot antenna arrays.

Polarization conversion surfaces (PCMs) are widely used in RCS reduction [1-4]. Some multilayer PCMs are proposed to obtain wider bandwidth. But the multilayer PCM is too complicated, which is not conducive to processing; single-layer PCM is more suitable for actual processing and application. A single-layer fishbone-shaped PCM unit is proposed [5]. The chessboard PCM array composed of it can reduce RCS by $10 \mathrm{~dB}$ from 5 to $17.5 \mathrm{GHz}$; RCS reduction ability is good, but the working frequency band is low, and the structure is complex, not suitable for high frequency. In recent years, some novel antennas integrated with metasurfaces are proposed to achieve a compact structure. A patch antenna based on ring-type concentric metasurface antenna (CMSA) with metal holes to the ground is designed to achieve reduction of RCS on the cylindrical carrier [6].
The ring-type metasurface reduces RCS from 4 to $8.5 \mathrm{GHz}$, but only the peak RCS reduction exceeds $10 \mathrm{~dB}$, the RCS reduction performance is relatively poor, and the concentric structure increases the plane area, which is not suitable for antenna arrays. In [7], the anisotropic metasurface is proposed to replace the patch antenna and behave as it directly, through proper connection of the metasurface units; the array achieves in-band RCS reduction with radiation performance kept. The proposed metasurface radiating array works from 3 to $3.3 \mathrm{GHz}$; the RCS reduction exceeds $10 \mathrm{~dB}$ at the peak. The structures of the two documents mentioned above have low working bands and only a few frequency points have reduced RCS by more than $10 \mathrm{~dB}$; the RCS reduction capacity is not good enough. At present, there is not much research on $\mathrm{W}$-band polarization conversion surface. A Venus-shaped PCM unit is proposed [8], which achieves a polarization conversion rate of $90 \%$ from 95.7 to 99.3 GHz; the 1-bit coded PCM array with the unit randomly distributed can manipulate the backscattered RCS pattern shape. The PCM works in W-band; the processing of structures working at such a high frequency is difficult; errors are easily introduced. In addition, its working frequency band is relatively narrow and the RCS reduction ability is insufficient. 
In this paper, a W-band single-layer capsule-shaped PCM unit is designed. The PCM is simple in shape and easy to process, and its polarization conversion rate (PCR) exceeds $97 \%$ in the W-band; the chessboard array designed on the basis of the proposed PCM unit achieves an RCS reduction of more than $10 \mathrm{~dB}$ in the $\mathrm{W}$-band. Placing the PCM array on a circularly polarized sequentially rotated slot antenna array, the RCS of the slot antenna array with PCM array is reduced by more than $10 \mathrm{~dB}$ from 40 to $119 \mathrm{GHz}$ with the radiation characteristics of the antenna array preserved; the relative bandwidth $(-10 \mathrm{~dB})$ reaches $96.3 \%$. The measurement results show that the $-10 \mathrm{~dB}$ impedance bandwidth of the antenna array is $35.4 \%(70-89 \mathrm{GHz})$, and the $3 \mathrm{~dB}$ axial ratio bandwidth is $13.8 \%(70-87 \mathrm{GHz})$.

\section{PCM Array Design}

According to the literatures, it can be known that the periodic diagonal metal structure can realize polarization rotation. Among them, the most basic structure is rectangle. The structure of the novel PCM unit proposed in this paper is shown in Figure 1. The unit is composed of PCM metal patch, dielectric substrate, and ground metal layer. The period is $t=2 \mathrm{~mm}$, the metal patch consists of a rectangle (length $l=1.2 \mathrm{~mm}$ and width $w=0.6 \mathrm{~mm}$ ) and two semicircles with the diameter $w$. The overall shape is similar to a capsule; the metal patch is placed along the diagonal direction. The dielectric substrate is Rogers RT5880 with a thickness of $0.508 \mathrm{~mm}$.

As shown in Figure 1, the diagonal structure is irradiated by the y-polarized plane wave, and the incident field $\vec{E}_{i}$ can be decomposed into two orthogonal vectors $\vec{E}_{i m}$ and $\vec{E}_{i n}$. The surface current of this capsule-shaped patch is shown in Figure 2(a). We can see that almost all the current flows along the n-axis. Therefore, the PCM can be equivalent to a PEC surface, and the reflected field has the same amplitude and opposite phase as the incident field, $\vec{E}_{r n}=-\vec{E}_{i n}$. Since there is little current flowing along the m-axis, the PCM can be seen as a high impedance surface; the reflected field and the incident field have the same amplitude and phase, $\vec{E}_{r m}=\vec{E}_{i m}$. The two orthogonal vectors $\vec{E}_{r m}$ and $\vec{E}_{r n}$ form the reflected field $\vec{E}_{r}$ which is in x-axis direction and orthogonal to the incident field, so a $90^{\circ}$ polarization rotation is obtained.

For comparison, the surface current of the basic rectangle PCM is shown in Figure 2(b). Most of the current flows along the $n$-axis, while at a right angle, a part of current flows in other directions, which will affect the effect of polarization rotation. The cause is that the sudden change at the right angle leads to the discontinuity of the electric field. Therefore, the capsule-shaped PCM proposed in this paper can eliminate this sudden change and achieve better polarization rotation effect, as shown in Figure 2(a). In addition, due to the gradual change of structure, the capsule-shaped PCM has a wider bandwidth.

The polarization conversion rate can be used to judge the polarization conversion performance of the PCM unit. Define the copolarization reflection coefficient $r_{\text {yy }}$ and cross-

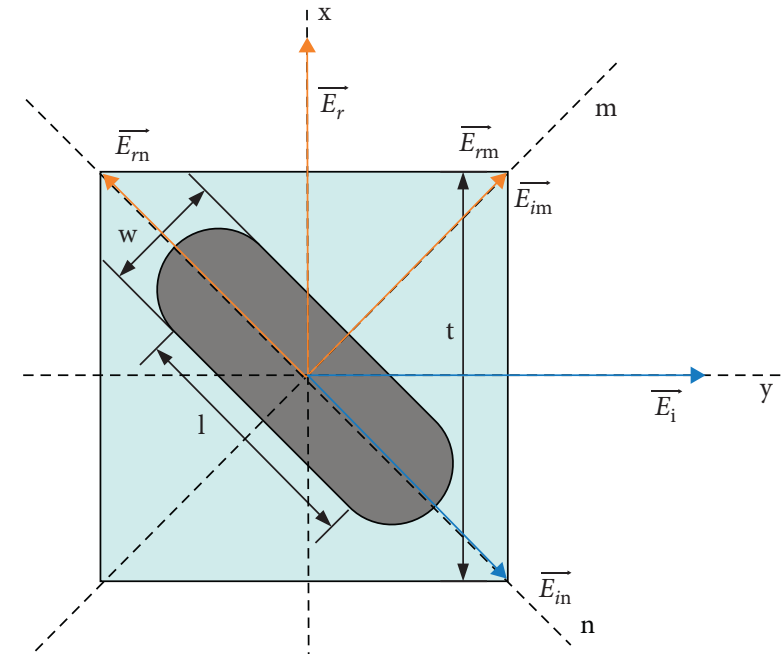

FIgURE 1: The structure of the capsule-shaped PCM unit.

polarization reflection coefficient $r_{\mathrm{xy}}$, as shown in the following equations:

$$
\begin{aligned}
& r_{y y}=\frac{\left|E_{r y}\right|}{\left|E_{i y}\right|}, \\
& r_{x y}=\frac{\left|E_{r x}\right|}{\left|E_{i y}\right|} .
\end{aligned}
$$

In the formula, $E_{r x}$ represents the electric field of the incident wave polarized in the $x$ direction, and $E_{\text {iy }}$ represents the electric field of the incident wave polarized in the $y$ direction. Correspondingly, $E_{\mathrm{rx}}$ represents the electric field of the reflected wave polarized in the $x$ direction, and $E_{\text {ry }}$ represents the electric field of the reflected wave polarized in the $y$ direction. The definition of polarization conversion rate $(\mathrm{PCR})$ can be given in the following equation:

$$
\mathrm{PCR}=\frac{r_{x y}^{2}}{\left(r_{x y}^{2}+r_{y y}^{2}\right)} \text {. }
$$

Use electromagnetic simulation software CST to simulate the designed capsule and rectangular PCM units; $r_{\mathrm{yy}}$ and $r_{\mathrm{xy}}$ of the two structures are obtained, as shown in Figure 3(a). For the capsule PCM, the copolarization reflection coefficient $r_{\mathrm{yy}}$ is less than $-10 \mathrm{~dB}$ and the crosspolarization reflection coefficient $r_{\mathrm{xy}} \approx 1$ in the band of 49-118.7 GHz. However, the rectangular PCM only obtained a sufficiently small copolarization reflection coefficient in a narrower band. Figure 3(b) shows the polarization conversion ratios (PCR) of the two PCM units. The capsule PCM unit achieves $90 \%$ PCR in $82.7 \%$ relative bandwidth, and the PCR exceeds $97 \%$ in the whole $\mathrm{W}$-band. The rectangular PCM achieves 90\% PCR in the band of 77.3-118 GHz, which cannot cover the entire $\mathrm{W}$-band, and its in-band polarization rotation effect is not satisfactory. In a word, the proposed capsule PCM has the wider band and higher polarization conversion rate than the rectangular PCM. 


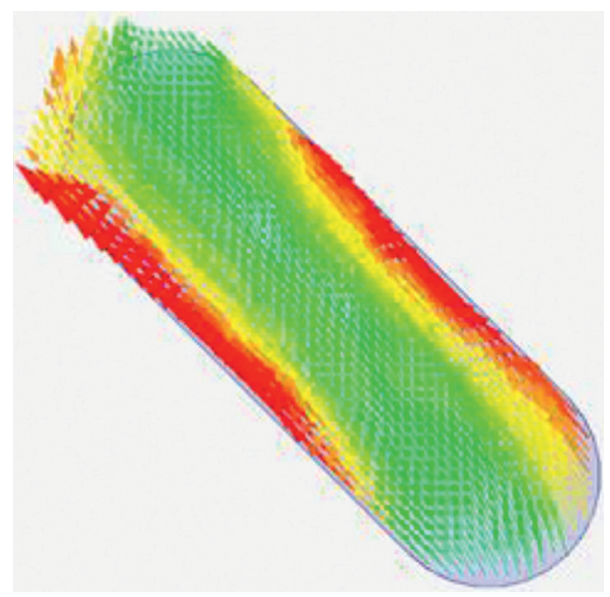

(a)

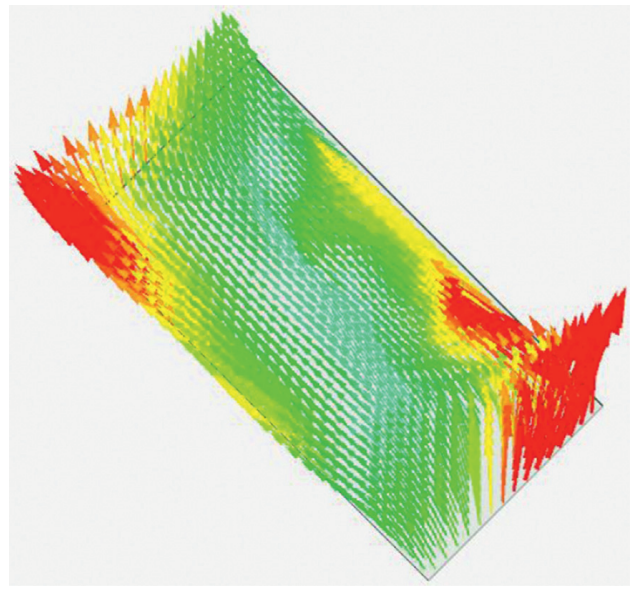

(b)

FIgURe 2: Surface current distribution of PCM unit. (a) Capsule. (b) Rectangle.

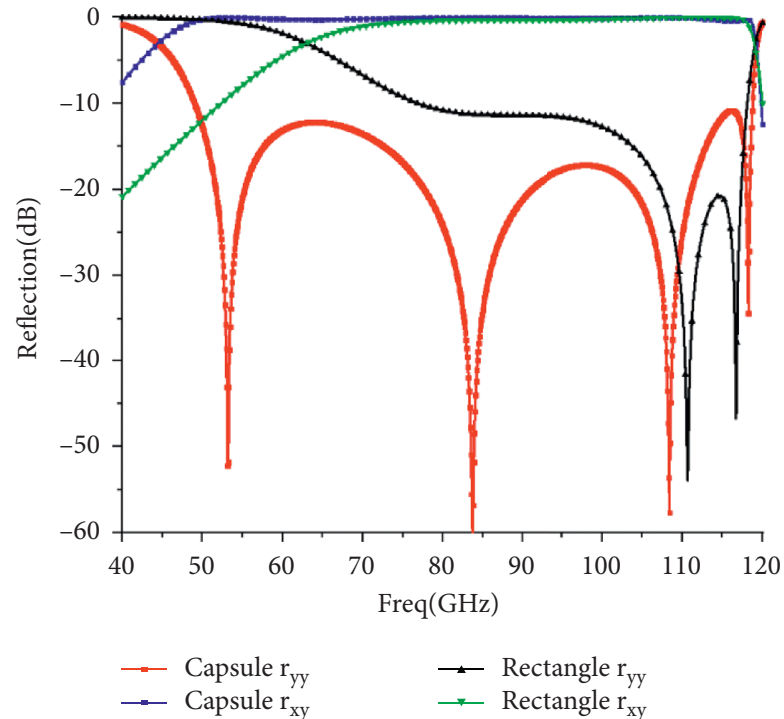

(a)

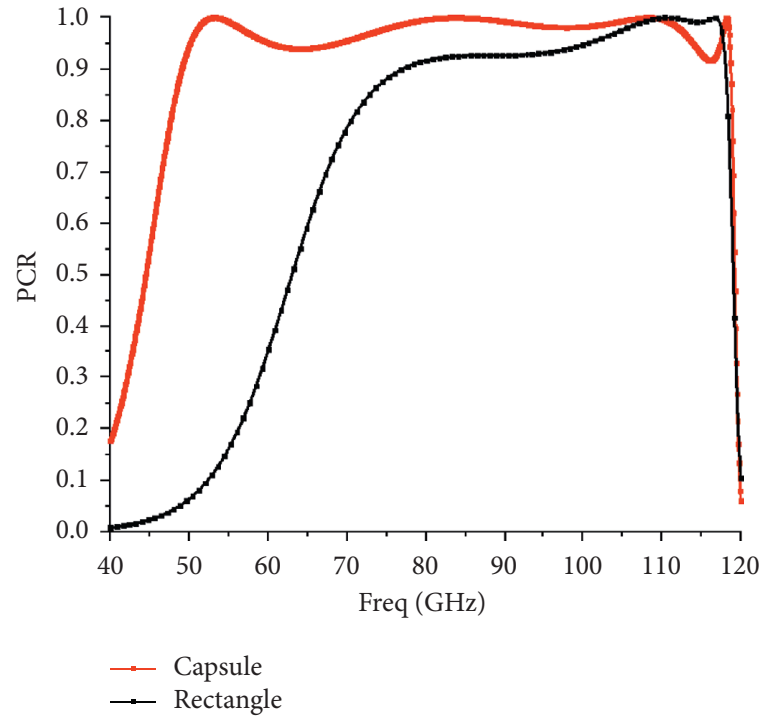

(b)

Figure 3: Comparison of capsule and rectangular PCM. (a) Reflection coefficient. (b) PCR.

The capsule PCM units are periodically arranged in a chessboard array, as shown in Figure 4. It should be noted that the circumference of the PCM array must be greater than 10 times the wavelength of the lowest operating frequency to ensure that it is in the optical scattering region. The PCM array is composed of four subarrays that rotate in order. Each subarray has 36 PCM units, the distance between the units is $2 \mathrm{~mm}$, and the size of the entire array is $24 \mathrm{~mm} \times 24 \mathrm{~mm}$. The simulated result of the RCS reduction of PCM array is plotted in Figure 5. According to the simulation results, the PCM array can achieve an RCS reduction of more than $10 \mathrm{~dB}$ in the range of $48-118 \mathrm{GHz}$, with a relative bandwidth of $84.3 \%$.

Figure 6 plots the three-dimensional bistatic RCS patterns of the PCM array and the PEC surface at $82 \mathrm{GHz}$. When the plane wave is incident vertically, the main reflection lobes of PCM appear in four quadrants, that is, they scatter in four different directions, while the reflection lobes of PEC concentrate in the incident direction. Therefore, the PCM surface can disperse the reflection and reduce the RCS.

The RCS patterns of the principal plane $\left(\mathrm{phi}=0^{\circ}, \mathrm{phi}=90^{\circ}\right)$ and the diagonal plane $\left(\mathrm{phi}=45^{\circ}\right.$, phi $=135^{\circ}$ ) at $82 \mathrm{GHz}$ are shown in Figures 7(a) and 7(b), respectively. On the principal plane, the maximum RCS scattering of the PCM array is about $30 \mathrm{~dB}$ lower than that of the PEC. On the diagonal, the maximum RCS scattering of the PCM array is $5.76 \mathrm{~dB}$ lower than that of the PEC, and the maximum RCS of the PCM array is at theta $= \pm 7^{\circ}$, which is consistent with the results in Figure 6 .

\section{Design and Measurement of the Low-RCS Slot Array Antenna with PCM}

3.1. Antenna Structure. In this section, a circularly polarized slot antenna array is designed, which shares a grounded metal plane with the PCM array proposed in the previous 


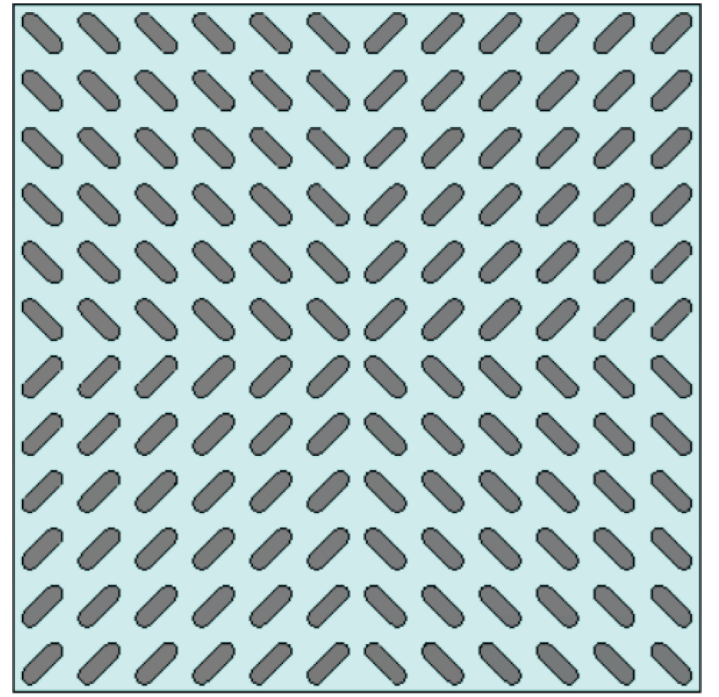

Figure 4: PCM array.

section; the antenna array uses the same substrate Rogers RT5880 as the PCM array; the thickness is $0.127 \mathrm{~mm}$. The slots are etched on the ground metal layer and fed by the feeding network at the bottom layer. The overall antenna structure is shown in Figure 8.

The design of the antenna's feeding network is shown in Figure 9; sequential rotated feeding is used to realize circularly polarized radiation [9-15]. It can be seen from Figure 9 that the 64 rectangular slot radiating elements and their feeding network are divided into 4 subarrays, the four subarrays are connected end to end and can be copied from one of the subarrays rotating around the center point, and each is fed by a 1 to 16 power divider. The four subarrays are fed in series to obtain equal amplitudes. Compared with the traditional array, this feeding structure has fewer microstrip lines and thereby reduces conduction loss, improves radiation efficiency, and increases gain bandwidth. Three $\mathrm{U}$-shaped lines are added to the feedlines at the input of the subarrays to provide a $90^{\circ}$ phase difference among the four subarrays. Therefore, the direction of the radiated electric field vector always changes with time, which forms a circularly polarized radiation.

In the designed multilayer structure, the arrangement direction of the slot antenna is the same as that of the PCM. This arrangement allows the four subarrays to obtain a natural sequential rotated relationship. This paper studies the influence of different arrangements of slots and PCMs on the antenna radiation. As shown in Figure 10, a simple ideal four-element array is designed to compare the antenna radiation performance of different arrangements. The overlapped and staggered arrangements of slots and PCMs were simulated; the simulated results are shown in Figures 11 and 12. Compared with the radiation pattern without PCM, it can be seen in Figure 11 that the overlapped arrangement increases the forward radiation gain of the slot antenna and weakens the backward radiation gain, which is not undesirable, and the staggered arrangement makes the main lobe of the E-plane deflect in the direction of the PCM,

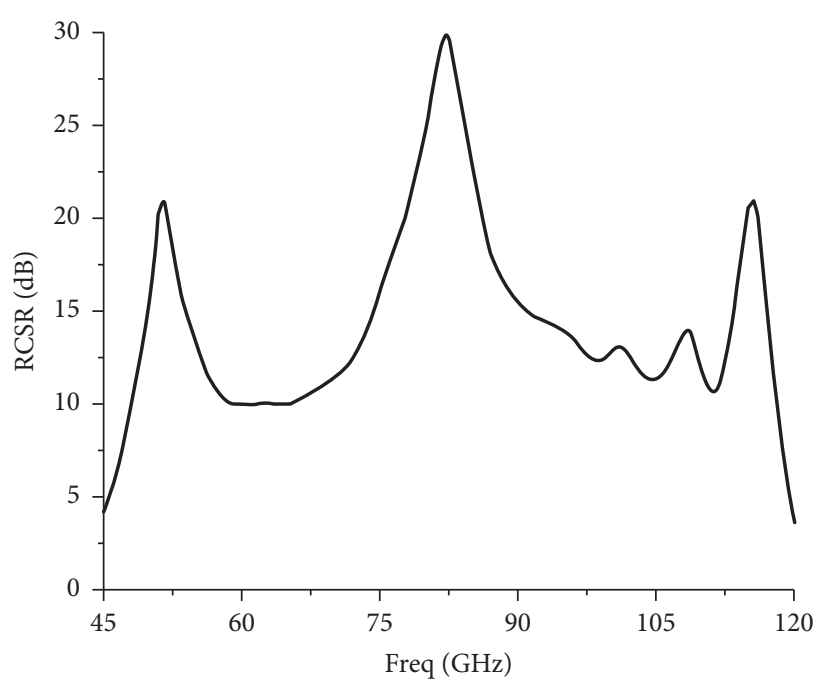

FIgURE 5: RCS reduction of capsule PCM array.

which has an adverse effect on the construction of subsequent arrays. In addition, as shown in Figure 12, compared with the array without PCM, the axial ratio of the overlapped arrangement is more fitting; its axial ratio is close to $0 \mathrm{~dB}$ at the central operating frequency of the antenna. However, the axial ratio of the staggered arrangement is relatively worse in the range of $79 \mathrm{G}$ to $86 \mathrm{G}$, which is exactly near the antenna central operating frequency. Since the feed is ideal, the axial ratio of the two arrangements is less than $3 \mathrm{~dB}$ from $75 \mathrm{GHz}$ to $90 \mathrm{GHz}$. Therefore, considering the effects on the antenna radiation pattern, axial ratio, and array performance, this paper chooses the overlapped arrangement of slots and PCMs.

The method of using sequential rotated feeding network to achieve circular polarization was first proposed by Tasuku [16]; in a sequential rotated array, each subarray is physically rotated by $90^{\circ}$ (360 divided by 4 subarrays) from the previous subarray. According to Tasuku's analysis, the far-field electric field vector of each subarray can be expressed as a linear combination of two orthogonal element vectors, and each subarray is fed with a different phase, assuming that the electric field vectors of the subarrays are equal in magnitude; after integration, the two orthogonal vectors in the total electric field vector of the array have equal magnitude coefficients. This means that the sequential rotated array provides good circularly polarized radiation; sequential rotated array can greatly reduce cross-polarization including noncenter frequencies; therefore, a circularly polarized array with expanded bandwidth can be obtained.

After optimization, the dimension drawing and parameters of the antenna are shown in Figure 13 and Table 1. The slots etched on the ground layer are rectangles of $1.25 \mathrm{~mm} \times 0.4 \mathrm{~mm}$; the slot unit uses a microstrip line to feed centrally; the characteristic impedance of the main feeding microstrip line is $106 \Omega$. The entire feeding network is matched to a $50 \Omega$ port through a section of $1 / 4 \lambda$ impedance transformation line; then, it is converted into a waveguide port by a microstrip-waveguide conversion structure. Figure 14 shows a physical prototype of the overall structure. 


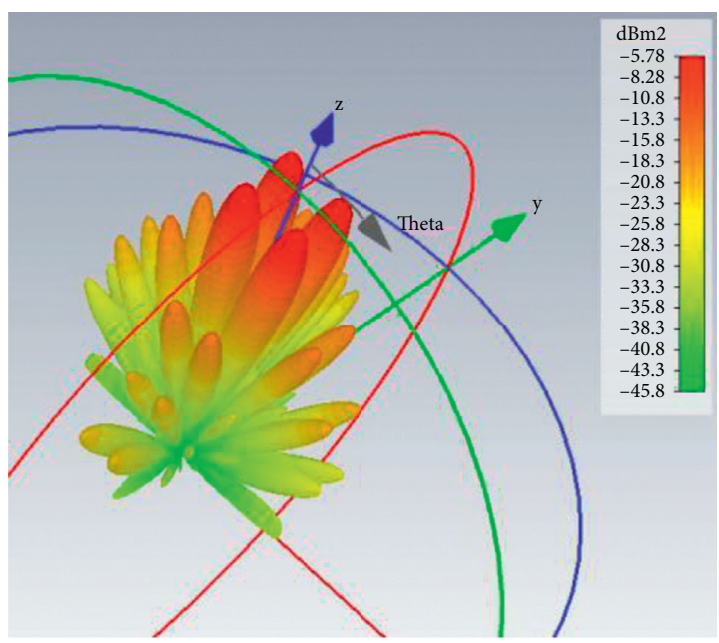

(a)

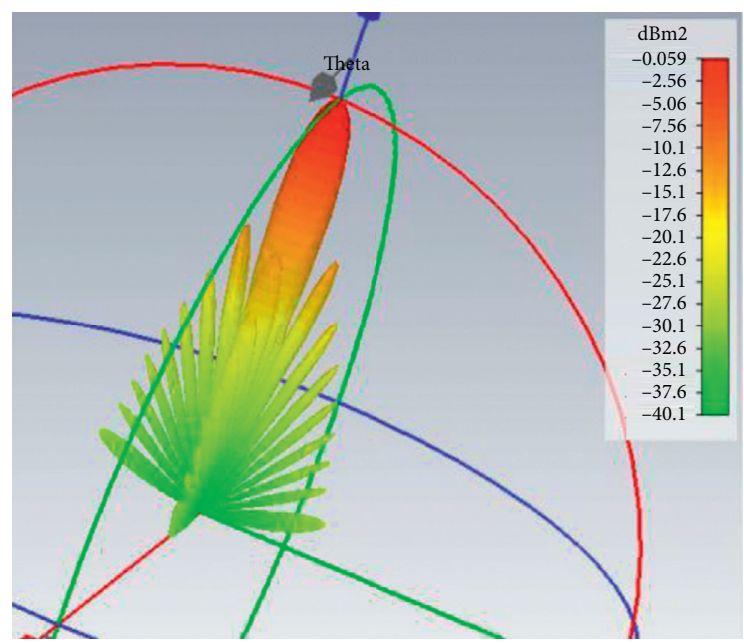

(b)

Figure 6: RCS pattern at $82 \mathrm{GHz}$. (a) PCM. (b) PEC.

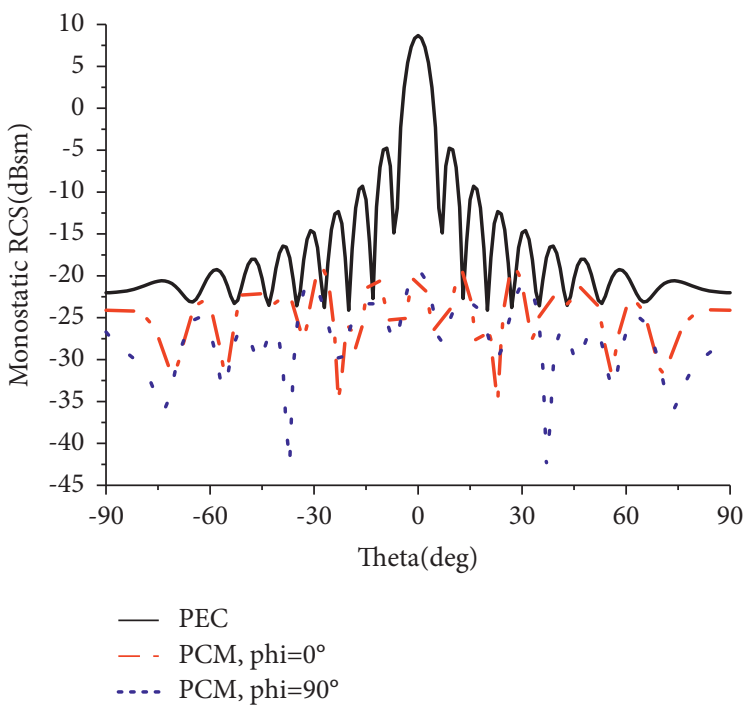

(a)

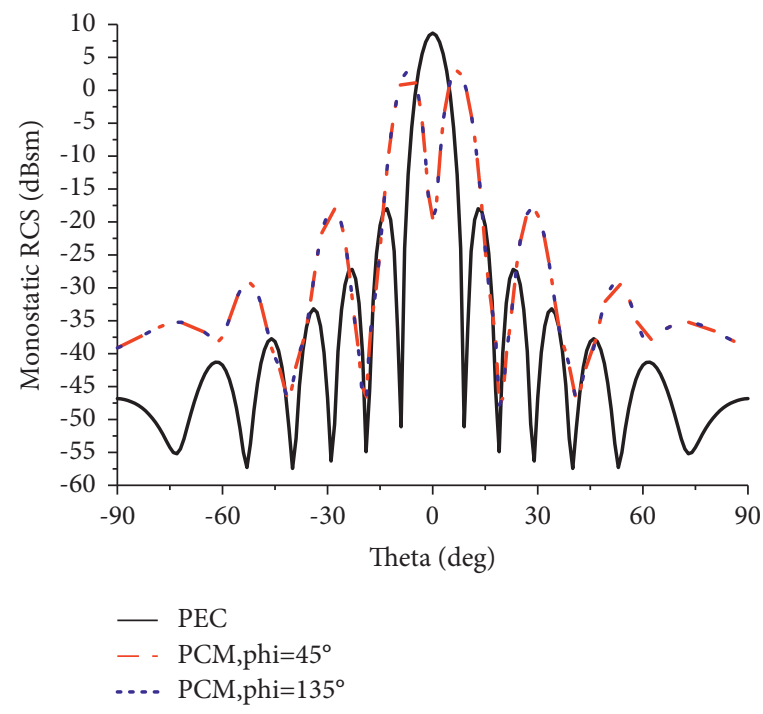

(b)

Figure 7: Comparison of RCS pattern of PCM and PEC at $82 \mathrm{GHz}$. (a) phi $=0^{\circ}$ and $90^{\circ}$. (b) phi $=45^{\circ}$ and $135^{\circ}$.

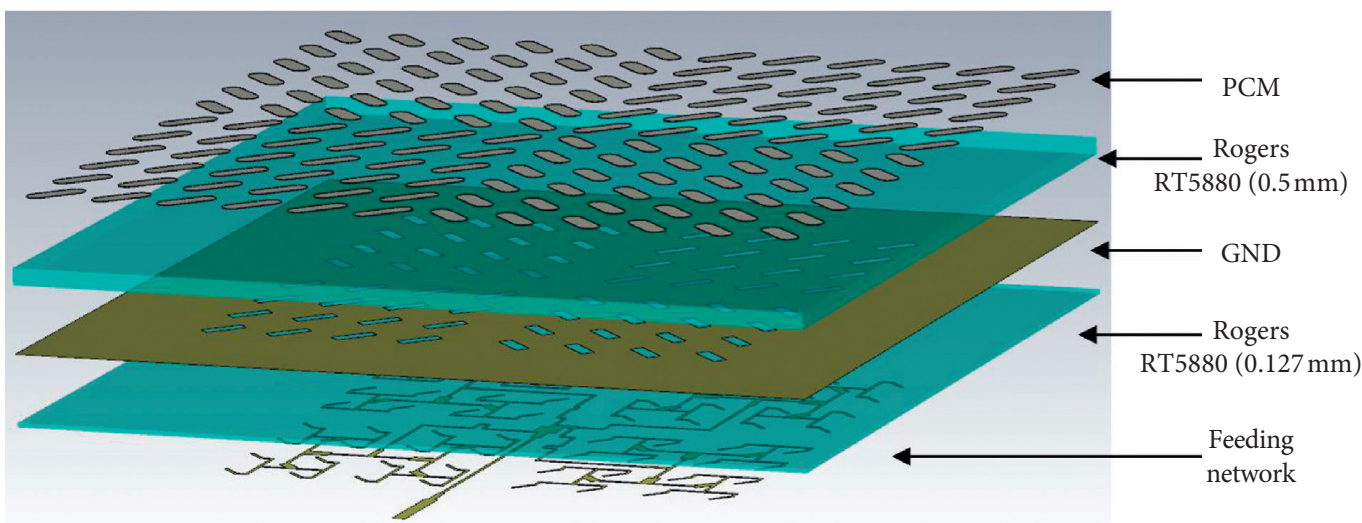

FIGURE 8: Structure of slot antenna array with RCS reduction. 


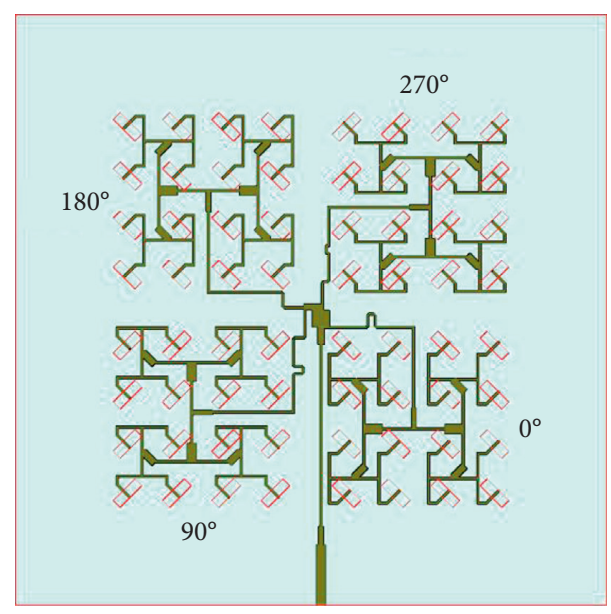

FIgURE 9: Structure of the feeding network.

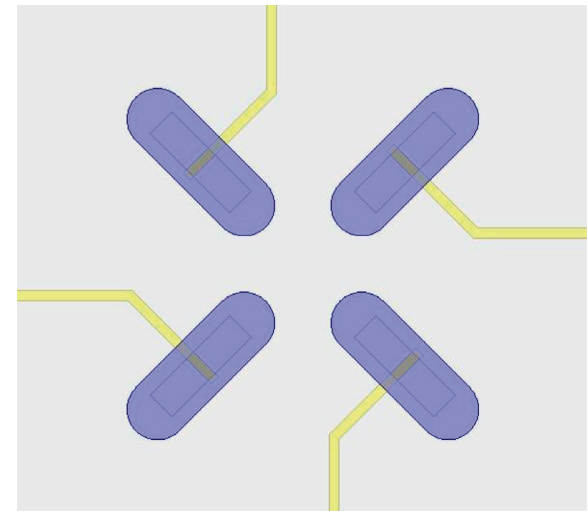

(a)

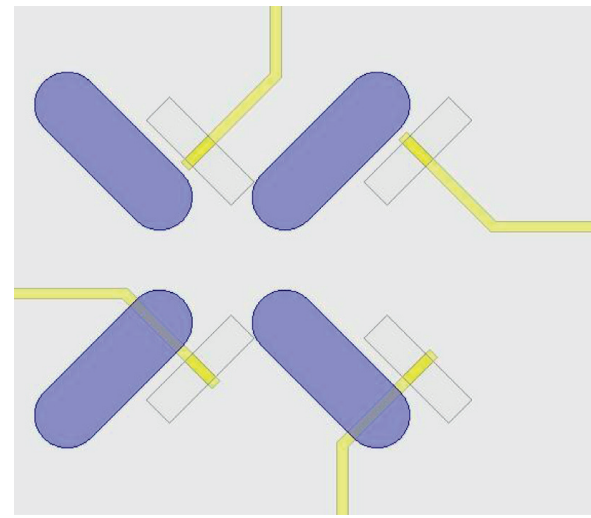

(b)

Figure 10: Different arrangements of slots and PCMs. (a) Overlapped. (b) Staggered.

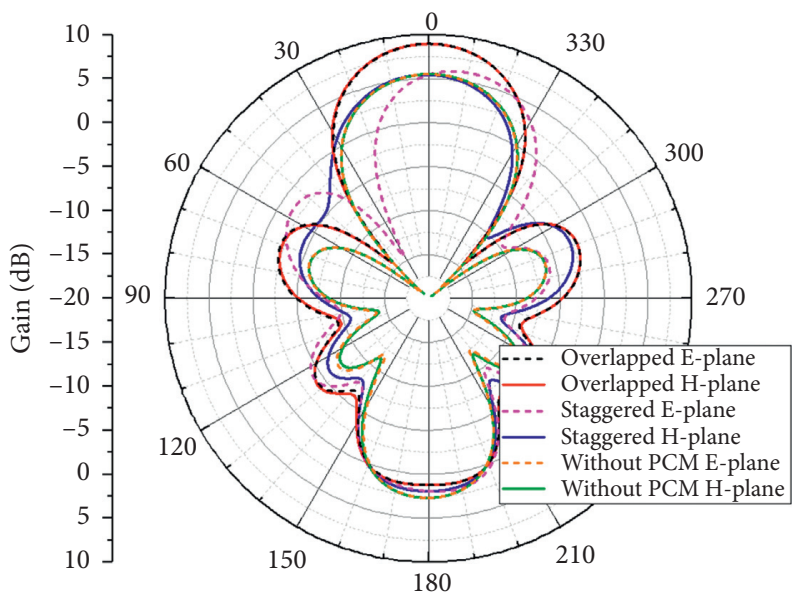

FIgURE 11: The pattern of different arrangements of slots and PCMs.

3.2. Simulated and Measured Radiation Characteristics. Based on the previous analysis and design, this section presents the simulated and measured results of the radiation characteristics of slot antenna array with RCS reduction. The simulated and measured results of the antenna's return loss are shown in Figure 15. It can be seen that the measured results 


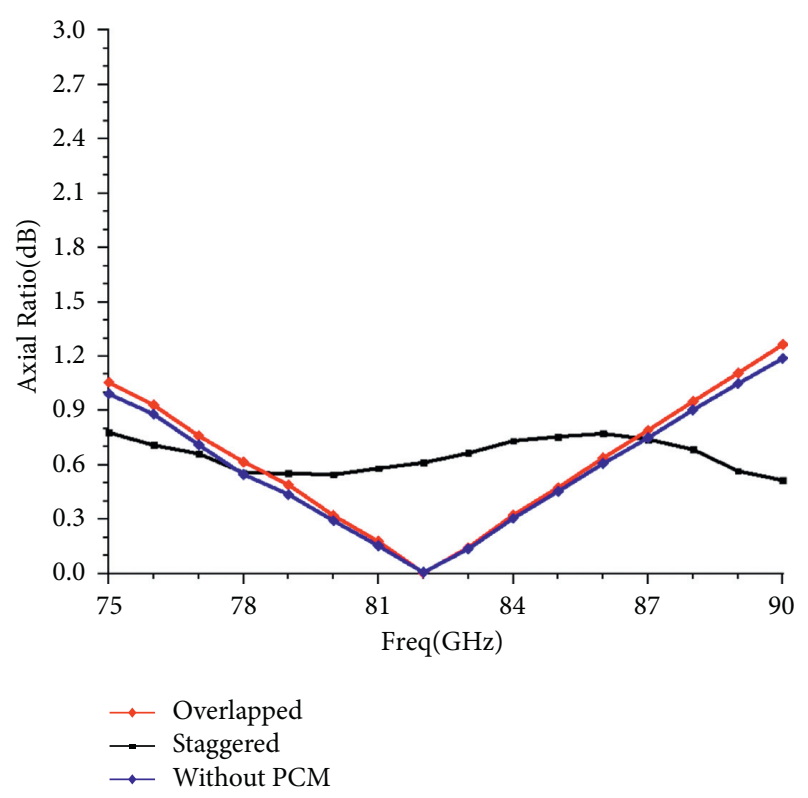

FIGURE 12: The axial ratio of different arrangements of slots and PCMs.

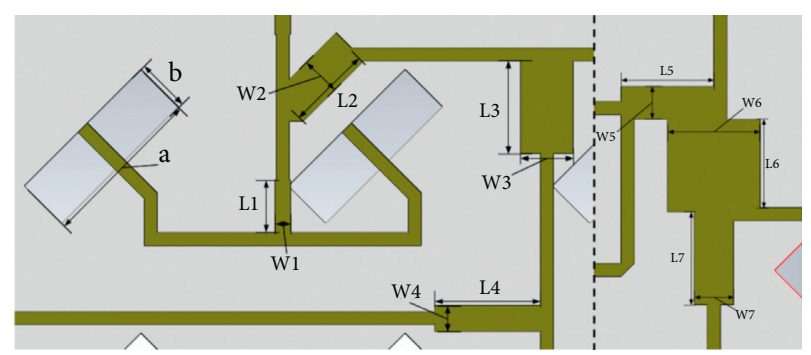

FIgURE 13: Dimension drawing of the proposed antenna.

TABLE 1: Design parameters of the proposed antenna.

\begin{tabular}{lccc}
\hline Parameter & Value $(\mathrm{mm})$ & Parameter & Value $(\mathrm{mm})$ \\
\hline a & 1.25 & L7 & 0.7 \\
b & 0.4 & W1 & 0.12 \\
L1 & 0.4 & W2 & 0.3 \\
L2 & 0.5 & W3 & 0.4 \\
L3 & 0.7 & W4 & 0.2 \\
L4 & 0.8 & W5 & 0.25 \\
L5 & 0.7 & W6 & 0.7 \\
L6 & 0.7 & W7 & 0.3 \\
\hline
\end{tabular}

gain good agreement with the simulated results. The relative impedance bandwidth $(-10 \mathrm{~dB})$ of the antenna is $35.4 \%$, and the working frequency band is $70-89 \mathrm{GHz}$, which can be covered by the working frequency band of the PCM array.

Figures 16(a) and 16(b) respectively show the simulated and measured results of the left-hand and right-hand circular polarization patterns of the antenna's far-field E-plane and H-plane at $82 \mathrm{GHz}$; the measured results are in good agreement with the simulated results. The main lobe gain of the antenna array is $14.9 \mathrm{~dB}$, the $3 \mathrm{~dB}$ beam width is $10.8^{\circ}$, and the first sidelobe level is $-10 \mathrm{~dB}$. Due to the complexity of the structure, the proposed antenna array does not have low sidelobe processing; another

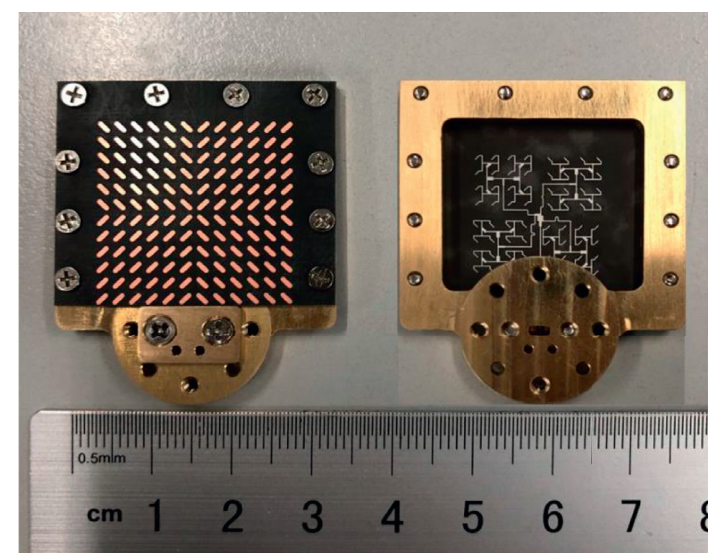

FIgURE 14: Prototype of antenna array with RCS reduction.

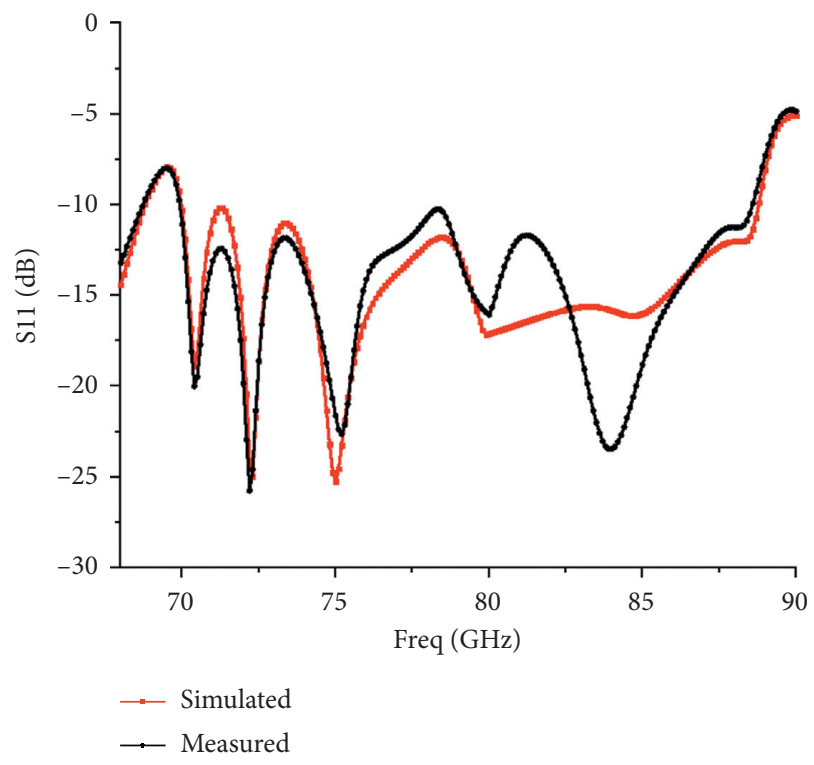

Figure 15: Simulation and measurement results of the return loss of the antenna array.

reason for the unsatisfactory sidelobe is that the distance between the subarrays is extended due to the existence of the feeding network. It can be seen from the figure that the right-handed polarization gain is obviously greater than the left-handed polarization gain; the gain of the left-handed polarization main lobe is $10 \mathrm{~dB}$ lower than that of the right-handed polarization, forming a good right-handed circularly polarized radiation.

Figure 17 shows the simulated and measured results of the axial ratio of the antenna array. The measured results are in good agreement with the simulated results. The axial ratio is less than $3 \mathrm{~dB}$ from 76.3 to $87 \mathrm{GHz}$, and the axial ratio bandwidth is $13.8 \%$; it shows that the antenna array radiates a good circularly polarized wave.

3.3. Simulated and Measured Scattering Property. The simulated and measured results of the RCS reduction are shown in Figure 18. As can be seen from the figure, the measured results are in good agreement with the simulated results. The RCS 


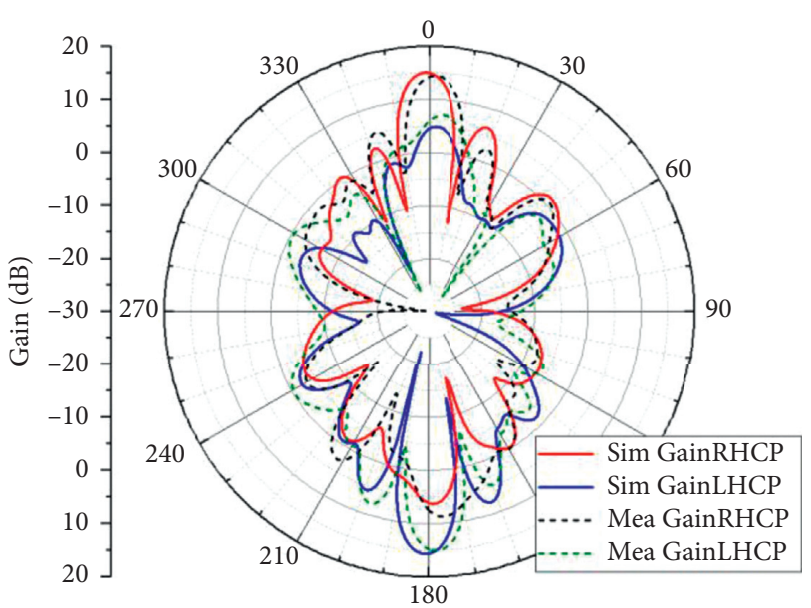

(a)

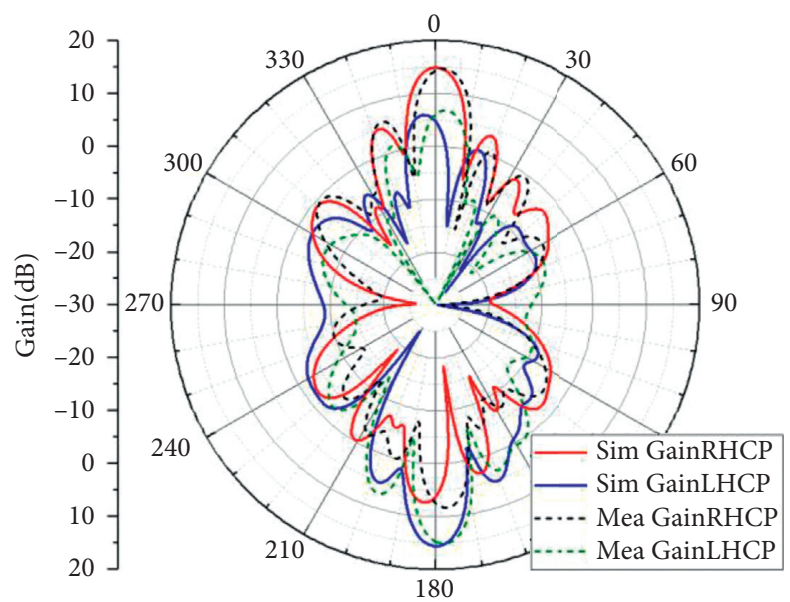

(b)

Figure 16: Simulated and measured results of antenna pattern. (a) E-plane. (b) H-plane.

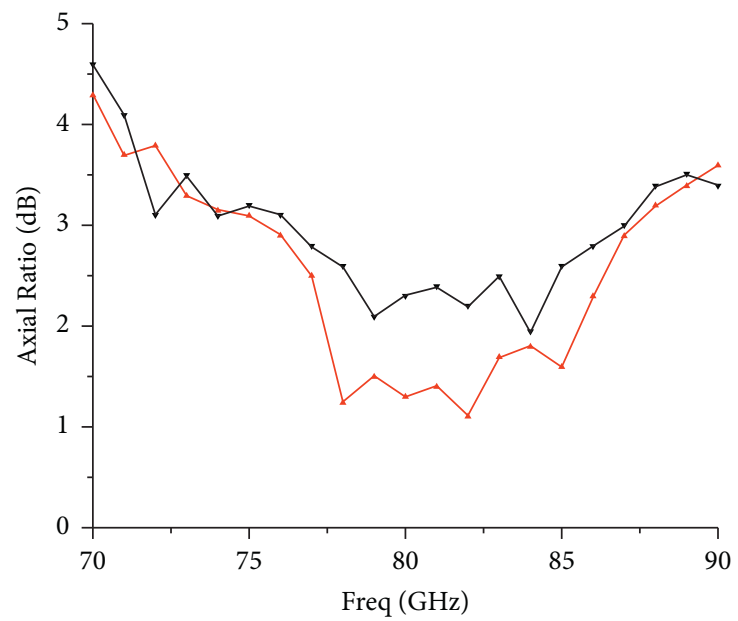

$\rightarrow$ Simulated

Measured

Figure 17: Simulation and measurement results of the axial ratio of the antenna array.

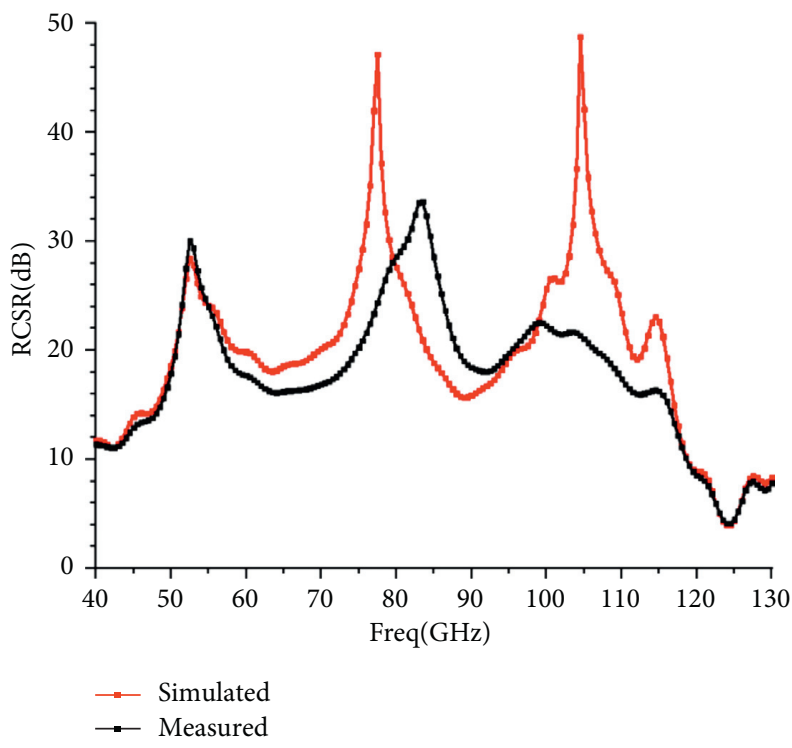

FIGURE 18: Measurement results of RCS reduction. 
TABLE 2: Comparison of characteristics with other works.

\begin{tabular}{lccc}
\hline Ref. & Frequency band & 10 dB RCSR bandwidth (\%) & Structure complexity \\
\hline$[5]$ & $\mathrm{C}-\mathrm{Ku}$ & 111 & Complicated \\
{$[6]$} & $\mathrm{C}-\mathrm{X}$ & $<20$ & Simple \\
{$[7]$} & $\mathrm{S}$ & $<10$ & Complicated \\
{$[8]$} & $\mathrm{W}$ & $<5$ & Complicated \\
This work & $\mathrm{W}$ & 99.4 & Simple \\
\hline
\end{tabular}

reduces more than $10 \mathrm{~dB}$ from 40 to $119 \mathrm{GHz}$, and the relative bandwidth reaches $99.4 \%$, covering the entire $\mathrm{W}$-band. The measured result of RCS reduction does not match the simulated result very well at high frequency, which may be caused by processing error.

In order to show the advantages of the structure designed in this paper, a comparison table is given below to illustrate the difference of the structure proposed with other recent works. As shown in Table 2, the PCM array proposed in this paper can work in ultra-high frequency bands, such as W-band, and in such a high frequency band, the proposed PCM array achieves ultra-wideband with a relative bandwidth close to $100 \%$. It is undeniable that the structure designed in this article shows great advantages in terms of working characteristics and application prospects. In addition, the capsule-shaped PCM unit designed in this paper greatly reduces the processing difficulty and error with its simple structure. The sequential rotation array structure also makes it very suitable for various antenna arrays.

\section{Conclusion}

A novel capsule-shaped PCM structure is proposed and has more than 97\% PCR in the whole W-band. The chessboard PCM has the wideband RCS reduction, and the performance of the slot antenna array is almost unaffected due to the consistency of the sequential rotation structure. The measurement results show that the RCS of the slot antenna array with PCM array is reduced by more than $10 \mathrm{~dB}$ from 40 to $119 \mathrm{GHz}$; the relative bandwidth $(-10 \mathrm{~dB})$ reaches $96.3 \%$. The $-10 \mathrm{~dB}$ impedance bandwidth of the antenna array is $35.4 \%(70-89 \mathrm{GHz})$, and the $3 \mathrm{~dB}$ axial ratio bandwidth is $13.8 \%(70-87 \mathrm{GHz})$. Because of its simple structure, easy processing, and low cost, the PCM will have wide applications in high frequency band.

\section{Data Availability}

The data used to support the findings of this study are included within the article.

\section{Conflicts of Interest}

The authors declare that there are no conflicts of interest regarding the publication of this paper.

\section{References}

[1] S. J. Li, X. Y. Cao, L. M. Xu et al., "Data from air force engineering university provide new insights into algorithms (Ultra-broadband reflective metamaterial with RCS reduction based on polarization convertor, information entropy theory and genetic optimization algorithm)," Science Letter, vol. 5, 2016.

[2] H. Chen, H. Ma, and S. Qu, "Ultra-wideband polarization conversion metasurfaces," in Proceedings of 3rd Asia-Pacific Conference on Antennas and Propagation, APCAP, Harbin, China, July 2014.

[3] Q. Zheng, C. Guo, and J. Ding, "Wideband and low RCS circularly polarized slot antenna based on polarization conversion of metasurface for satellite communication application," Microwave and Optical Technology Letters, vol. 60, no. 3 , pp. 679-685, 2018.

[4] X. Luo, Q. Zhang, and Y. Zhuang, "Tai-Chi-Inspired pancharatnam-berry phase metasurface for dual-band RCS reduction," in Proceedings of the IEEE International Symposium on Antennas and Propagation \& USNC/URSI National Radio Science Meeting, July 2017.

[5] Y. Liu, K. Li, Y. Jia, Y. Hao, S. Gong, and Y. J. Guo, "Wideband RCS reduction of a slot array antenna using polarization conversion metasurfaces," IEEE Transactions on Antennas and Propagation, vol. 64, no. 1, pp. 326-331, 2016.

[6] T. Li, H. Yang, Q. Li, L. Jidi, X. Cao, and J. Gao, "Broadband low-RCS and high-gain microstrip antenna based on concentric ring-type metasurface," IEEE Transactions on Antennas and Propagation, vol. 69, no. 9, pp. 5325-5334, 2021.

[7] H. Yang, T. Li, L. Xu et al., "Low in-band-RCS antennas based on anisotropic metasurface using a novel integration method," IEEE Transactions on Antennas and Propagation, vol. 69, no. 3, pp. 1239-1248, 2021.

[8] M. K. T. Al-Nuaimi and Y. He, "Cross polarization conversion and RCS reduction using venus-like metallic resonators," in Proceedings of the 2018 IEEE MTT-S International Wireless Symposium (IWS), pp. 1-4, Chengdu, China, May 2018.

[9] M. Yousefbeigi, A. Enayati, M. Shahabadi, and D. Busuioc, "Parallel-series feed network with improved G/T performance for high-gain microstrip antenna arrays," Electronics Letters, vol. 44, no. 3, pp. 180-182, 2008.

[10] B. Aljibouri, A. J. Sambell, and B. S. Sharif, "Application of genetic algorithm to design of sequentially rotated circularly polarized dualfeed microstrip patch antenna array," Electronics Letters, vol. 44, no. 12, pp. 1005-1006, 2008.

[11] M. N. Jazi and M. N. Azarmanesh, "Design and implementation of circularly polarized microstrip antenna array using a new serial feed sequentially rotated technique," IEEE Proceedings-Microwaves Antennas and Propagation, vol. 153, 2006.

[12] J. Huang, "A technique for an array to generate circular polarization with linearly polarized elements," IEEE Transactions on Antennas and Propagation, vol. 34, no. 9, pp. 1113-1124, 1986.

[13] P. S. Hall, J. S. Dahele, and J. R. James, "Design principles of sequentially fed, wide bandwidth, circularly polarised microstrip antennas," IEE Proceedings H Microwaves, Antennas and Propagation, vol. 136, no. 5, pp. 381-389, 1989. 
[14] S. Maddio, "A compact wideband circularly polarized antenna array for \$C\$ -band Applications," IEEE Antennas and Wireless Propagation Letters, vol. 14, pp. 1081-1084, 2015.

[15] K. D. Palmer, J. H. Cloete, and J. J. van Tonder, "Bandwidth improvement of circularly polarised arrays using sequential rotation," Antennas and Propagation Society International Symposium, vol. 19, pp. 135-138, 1992.

[16] T. Teshirgoi, M. Tanaka, and W. Chujo, "Wideband circularly polarized array antenna with sequential ratations and phase shift of elements," in Proceeding of the International Symposium on Antennas Propagation, pp. 136-139, Tokyo, Japan, August 1985. 